\title{
Eps8 vaccine exerts prophylactic antitumor effects in a murine model: A novel vaccine for breast carcinoma
}

\author{
YAN-JIE HE*, JING ZHOU*, TONG-FENG ZHAO*, LIANG-SHAN HU, \\ JING-YING GAN, LAN DENG and YUHUALI
}

Department of Hematology, Zhujiang Hospital, Southern Medical University, Guangzhou, Guangdong 510282, P.R. China

Received January 25, 2013; Accepted May 30, 2013

DOI: $10.3892 / \mathrm{mmr} .2013 .1514$

\begin{abstract}
Cancer vaccines are an effective way to prevent the occurrence of cancer. Epidermal growth factor receptor pathway substrate 8 (Eps8) is a novel tumor-associated antigen, which is overexpressed in the majority of tumor types. In the present study, the Eps8 protein was cloned and characterized, and its feasibility as an antitumor agent in murine breast carcinoma was investigated. The results revealed that the Eps8 protein increased the secretion of interleukin (IL)-12 in the culture supernatant of dendritic cells (DCs). The Eps8 protein-pulsed DCs induced significant cytotoxic T lymphocyte (CTL) responses, T-cell proliferation and a higher level of interferon (IFN) $-\gamma$ in the culture supernatant of the splenocytes ex vivo. Additionally, when the mice were immunized with the Eps8 vaccine, this resulted in a regression of $4 \mathrm{~T} 1$ breast tumors and significantly prolonged survival time in the tumor-bearing mice compared with that in the phosphate-buffered saline (PBS) control group. The Eps8 vaccine induced higher CTL responses in the splenocytes of mice vaccinated against the 4T1 cells; the ratio of $\mathrm{CD}^{+} / \mathrm{CD}^{+}$T cells was increased in the Eps8 group; and the percentage of $\mathrm{CD} 4{ }^{+} \mathrm{CD} 25^{+} \mathrm{FoxP} 3^{+}$regulatory $\mathrm{T}$ (Treg) cells in the Eps8 group was significantly lower compared with that of the PBS group. The results suggested that the Eps8 vaccine was able to stimulate antitumor effects against 4T1 breast cancer cells in vitro and in vivo, and it may provide a potential immunotherapeutic agent for the treatment of breast cancer.
\end{abstract}

\section{Introduction}

Breast cancer is the leading cause of cancer-related mortality in females worldwide and is the second leading cause of

Correspondence to: Dr Yuhua Li, Department of Hematology, Zhujiang Hospital, Southern Medical University, 253 Industry Road, Guangzhou, Guangdong 510282, P.R. China

E-mail: liyuhua2011gz@163.com

*Contributed equally

Key words: epidermal growth factor receptor pathway substrate 8, vaccine, cytotoxic T lymphocyte, Treg cells, immunotherapy cancer-related mortality (10.9\%), resulting in $14 \%$ of total fatalities due to cancer $(1,2)$. Traditional therapies for breast cancer (including surgery, radiotherapy and chemical treatment) may cause various adverse effects and have a high risk of relapse $(3,4)$. Therefore, improved technologies and treatment methods for breast cancer are required. The field of cancer vaccination is currently being investigated in clinical practice (5), and various antigens have been used in vaccine formulation. Several breast cancer antigens, such as human epidermal growth factor receptor 2 (HER2), mucin 1 (MUC1), human telomerase reverse transcriptase (hTERT), tumor protein 53 (p53) and cancer embryonic antigen (CEA), have been utilized in the preparation of these vaccines (6-9). The vaccines have either been tested alone, with antigenic epitopes or in combination with immune stimulants, including granulocyte-macrophage colony-stimulating factor (GM-CSF) and Bacillus Calmette-Gúerin (BCG). Presently, the vaccines have yet to demonstrate a protective outcome in clinical trials. Hence, novel vaccines capable of stimulating effective antitumor immune responses are required.

Epidermal growth factor receptor pathway substrate 8 (Eps8) is a $97-K D a$ protein, measuring 821 amino acids in length, which was originally identified as a substrate for epidermal growth factor receptor (EGFR) kinase (which mediates mitogenic signaling). The human Eps8 gene locus was mapped to chromosome 12q13.2. Computer-assisted analysis of the predicted amino acid sequence of Eps8 revealed a structural organization similar to that of a typical signaling molecule, including a putative $\mathrm{N}$-terminal PTB domain, a central SH3 domain and a C-terminal effector region. Two functional regions of Eps8 have been identified by structural-functional studies. The EGFR binding region is a binding surface for the juxtamembrane region of the EGFR, at amino acids 298-362. The other region is the C-terminal effector region, located at amino acids 648-821, which is sufficient to activate Rac and result in remodeling of the actin cytoskeleton $(10,11)$

Previous studies have demonstrated that Eps8 is overexpressed in the majority of cancer types, including oral squamous cell carcinoma and breast, cervical, colon, esophageal, pancreatic, thyroid and pituitary cancer, but is not expressed (or is poorly expressed) in normal tissues (12-19). Overexpressed Eps8 has also been demonstrated to be involved in numerous signaling pathways associated with the genesis, proliferation, migration, metastasis and chemoresistance of cancer, and is 
a marker of poor prognosis in cancer patients (12,14-21). The present study focused on whether Eps8 was able to block $\mathrm{T}$ regulatory (Treg) cells and induce antitumor immunity in a murine breast cancer model, as a novel protein vaccine.

\section{Materials and methods}

Mice and cell lines. Female BALB/c mice (age, 6-8 weeks) were purchased from the Laboratory Animal Center of Southern Medical University (Guangzhou, Guangdong, China). The experiments were conducted according to the guidelines of the China Council on Animal Care. The mice were allowed free access to food and water, and were maintained in specific pathogen-free conditions. The murine breast cancer cell line 4T1, was purchased from the Type Culture Collection of the Chinese Academy of Sciences, Shanghai, China. The murine breast cancer cell line, MA782, was provided by Dr. Yanqing Ding (Southern Medical University). All cells were maintained in Dulbecco's modified Eagle's medium (DMEM; Gibco-BRL, Carlsbad, CA, USA) supplemented with $10 \%$ fetal bovine serum (FBS; HyClone Laboratories, Inc., Logan, UT, USA), penicillin (100 units/ml; Sigma-Aldrich, St. Louis, MO, USA) and streptomycin (100 $\mu \mathrm{g} / \mathrm{ml}$; Sigma-Aldrich), at $37^{\circ} \mathrm{C}$ in a humidified atmosphere containing $5 \% \mathrm{CO}_{2}$. The study was approved by the ethics committee of Southern Medical University, Guangzhou, China.

Bacterial strains and vectors. BL21 E. coli cells and the

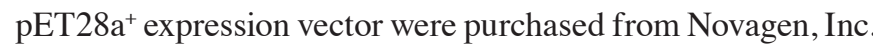
(Madison, WI, USA). The bacterial strains were grown at $37^{\circ} \mathrm{C}$ in Luria-Bertani (LB) broth or on LB agar plates, and kanamycin was added to the culture medium at a concentration of $50 \mu \mathrm{g} / \mathrm{ml}$ when required. Isopropyl- $\beta$-D-thiogalactopyranoside (IPTG, $1 \mathrm{mM}$ ) was added to the media as indicated.

PCR amplification and cloning of the Eps8 gene. Total RNA was extracted from MA782 cells using TRIzol reagent (Life Technologies, Inc., Gaithersburg, MD, USA). Based on the murine Eps8 gene sequence in the Genbank, a pair of PCR primers were designed (amino acids, 440-710). The primer sequences used were as follows: Forward: 5'-GCG GAA TTC CCG ATG CTG AAC TTC ATG-3', containing an EcoRI site; and reverse: 5'-ATA CTC GAG CCC GAT GGT CAG CCT CTG-3', containing a XhoI site. The reaction conditions were as follows: $94^{\circ} \mathrm{C}$ for $5 \mathrm{~min}, 94^{\circ} \mathrm{C}$ for $30 \mathrm{sec}, 60^{\circ} \mathrm{C}$ for $45 \mathrm{sec}$ and $72^{\circ} \mathrm{C}$ for $30 \mathrm{sec}$ (for a total of 35 cycles), and then $72^{\circ} \mathrm{C}$ for $10 \mathrm{~min}$. Following PCR amplification, the desired fragment was identified by agarose gel electrophoresis. Subsequent to purification, the PCR product and the pET28a vector were digested with $E c o$ RI and XhoI double enzymes, and linked by $\mathrm{T} 4$ DNA ligase, at $16^{\circ} \mathrm{C}$ for $16 \mathrm{~h}$. The sequence was analyzed by the sequencing laboratory of Invitrogen Corporation (Guangzhou, Guangdong, China). Following verification, the recombinant plasmid (pET28a-Eps8) was transformed into BL21 E. coli cells.

Eps8 protein expression, purification and western blot analysis. BL21 E. coli cells containing pET28a-Eps8 were inoculated in $5 \mathrm{ml} \mathrm{LB}$ broth (with $50 \mu \mathrm{g} / \mathrm{ml}$ kanamycin sulfate) and cultured overnight at $37^{\circ} \mathrm{C}$. The bacterial culture was grown in $100 \mathrm{ml} \mathrm{LB}$ broth to an OD600 of 0.8 , and was induced by $1 \mathrm{mM}$ IPTG for $4-5 \mathrm{~h}$ at $37^{\circ} \mathrm{C}$, harvested by centrifugation (5 min, 8,228 $\mathrm{x} \mathrm{g}$ at $4^{\circ} \mathrm{C}$ ), and then dispersed into a lysis buffer and sonicated. Nickel-nitrilotriacetic acid (Ni-NTA) agarose was used for $6 \mathrm{X}$ His-tagged protein purification, according to the manufacturer's instructions (Qiagen, Hilden, Germany). Endotoxin was removed from the protein using Pierce High Capacity Endotoxin Removal Resin (Thermo Fisher Scientific, Rockford, IL, USA), according to the manufacturer's instructions. The purified protein was boiled for $10 \mathrm{~min}$, and then loaded onto a $12 \%$ sodium dodecyl sulfate-polyacrylamide gel electrophoresis (SDS-PAGE) gel and electrophoresed. The samples were transferred onto a polyvinylidene difluoride membrane (Millipore, Billerica, MA, USA). The membrane was blocked by $5 \%$ skimmed milk for $1 \mathrm{~h}$, washed three times with phosphate-buffered saline (PBS)-Tween 20 (10 mM Tris-HCl, pH 7.5; $150 \mathrm{mM} \mathrm{NaCl} ; 0.05 \%$ Tween 20), and incubated with mouse anti-EPS8 monoclonal antibodies (BD Biosciences, San Diego, CA, USA) overnight at $4^{\circ} \mathrm{C}$. The membrane was then washed three times with PBS-Tween 20, and incubated with goat anti-mouse immunoglobulin G (Santa Cruz Biotechnology, Inc., Dallas, TX, USA) for $4 \mathrm{~h}$ at $37^{\circ} \mathrm{C}$. The immunoreactive bands were detected using the Amersham enhanced chemiluminescence (ECL) detection system and ECL hyperfilm (GE Healthcare, Waukesha, WI, USA), according to the manufacturer's instructions. The FluorChem FC2 imaging system was used to analyze the chemiluminescent blot (Alpha InnoTech Corp., San Leandro, CA, USA).

Generation of dendritic cells (DCs). Bone marrow cells were isolated from the femurs and tibias of 6- to 8-week-old BALB/c mice, and incubated in hypotonic lysis buffer $\left(9.84 \mathrm{~g} / 1 \mathrm{NH}_{4} \mathrm{Cl}\right.$, $1 \mathrm{~g} / 1 \mathrm{KHCO}_{3}, 0.1 \mathrm{mM}$ EDTA) for $5 \mathrm{~min}$ at $37^{\circ} \mathrm{C}$ in a water bath, to remove the red blood cells. The cells were washed twice with PBS buffer, and then plated in 6-well plates at a density of $1 \times 10^{6}$ cells $/ \mathrm{ml}\left(5 \times 10^{5}\right.$ cells/well) for $1 \mathrm{~h}$ at $37^{\circ} \mathrm{C}$, in RPMI-1640 medium with 10\% FBS. Non-adherent single cells were gently removed, and the adherent cells were cultured in $2 \mathrm{ml}$ RPMI-1640 with 10\% FBS, $50 \mathrm{ng} / \mathrm{ml}$ murine rGM-CSF (PeproTech, Inc., Rocky Hill, NJ, USA) and 20 ng/ml murine recombinant interleukin-4 (rIL-4; PeproTech, Inc.) per well for six days. For the induction of maturation, cultures of bone marrow-derived DCs (BM-DCs) were supplemented with $50 \mathrm{ng} / \mathrm{ml}$ murine tumor necrosis factor- $\alpha$ (TNF- $\alpha$; PeproTech, Inc.) for $24 \mathrm{~h}$ on the sixth day of the culturing process, prior to use $(22,23)$. The DCs were pulsed on day 7 with either Eps8 protein $(50 \mu \mathrm{g} / \mathrm{ml})$ or chicken egg ovalbumin (OVA; $50 \mu \mathrm{g} /$ ml; Alpha Diagnostic Intl Inc., San Antonio, TX, USA) for $24 \mathrm{~h}$, and the pulsing was terminated by medium replacement. Different groups of DCs were collected and washed three times with PBS buffer. The DCs were then incubated with the corresponding antibodies on ice for $30 \mathrm{~min}$. Flow cytometric analysis was performed on a fluorescence-activated cell sorter (FACSCalibur flow cytometer; Becton-Dickinson, Mountain View, CA, USA). The DCs were phenotyped with antibodies against complement component 3 receptor 4 subunit (CD11c), cluster of differentiation 80 (CD80), CD86 and major histocompatibility complex class II (MHC-II; BioLegend, San Diego, CA, USA). The negative controls consisted of DCs labeled with mouse Ig. 
Proliferation and cytotoxicity of splenocytes in vitro. The spleens were removed from 6- to 8-week-old BALB/c mice under sterile conditions. Subsequently, single-cell suspensions were prepared by pressing the spleens through a sterile 100-mesh metal sieve and filtrated using a 80- $\mu$ m nylon mesh. The lymphocytes from the suspension of the spleen cells $(6 \mathrm{ml})$ were isolated by Ficoll density gradient centrifugation at $514 \mathrm{x}$ g for $30 \mathrm{~min}$ at room temperature. The cells of the middle layer were carefully removed and washed twice with serumfree RPMI-1640 medium. The splenocytes were counted with a hemacytometer and plated in 6 -well plates $\left(5 \times 10^{6}\right.$ cells/well $)$, in 2-ml RPMI-1640 medium with heat-inactivated FBS and $20 \mathrm{ng} / \mathrm{ml}$ recombinant mouse IL-2 (24).

Proliferation of the splenocytes was determined by an MTT assay as previously described (25). Splenocytes were harvested and co-cultured with autologous DCs at a ratio of 5:1 in 96-well plates for $72 \mathrm{~h}$, and this ratio had been identified to be optimal (data not shown). The OD570 was read and the stimulation index was calculated by dividing the background mean absorbance from cultures with unstimulated splenocytes by the experimental mean absorbance from cultures with DC-stimulated splenocytes. All experiments were performed in triplicate and repeated three times.

The cytotoxic activity of the splenocytes was assessed by the CytoTox 96 Non-Radioactive Cytotoxicity Assay kit (Promega, Madison, WI, USA), according to the manufacturer's instructions. The splenocytes that were co-cultured with autologous DCs served as effector cells, while the 4T1 cells served as target cells. The target cell suspension was adjusted to $5 \times 10^{5}$ cells $/ \mathrm{ml}$ and the effector cell suspension was adjusted to $1 \times 10^{7}$ cells/ml, with RPMI-1640 medium (containing 5\% FBS), and the cells were plated in 96-well plates. The effector to target (E/T) cell ratio was 20:1. Wells containing only target cells or only effector cells with culture medium or $0.5 \%$ Triton X-100 served as spontaneous or maximal release controls. Following $4 \mathrm{~h}$ of incubation at $37^{\circ} \mathrm{C}$ and $5 \% \mathrm{CO}_{2}, 50 \mu \mathrm{l}$ supernatant was analyzed by the Model 680 microplate reader at a reference wavelength of $650 \mathrm{~nm}$ and a test wavelength of $450 \mathrm{~nm}$ (BioRad, Hercules, CA, USA). The percentage of specific lysis was calculated as follows: Specific lysis $(\%)=100 \mathrm{x}$ (experimental release - effector spontaneous release - target spontaneous release) / (target maximum release - target spontaneous release).

Enzyme-linked immunosorbent assay (ELISA) for evaluating interferon- $\gamma(I F N-\gamma)$ and $I L-12$. The supernatant of the splenocytes was collected at different time points (days 1, 3 and 5) and tested for the presence of IFN- $\gamma$ by ELISA, according to the manufacturer's instructions (Endogen, Inc., Cambridge, MA, USA). In order to detect the level of IL-12 that was secreted by the DCs, supernatants were collected at the aforementioned time points and analyzed for IL-12 p70, using the ELISA (Biosource, Camarillo, CA, USA).

Vaccination and inoculation protocol. The experiment was performed on 4- to 6-week-old female BALB/c mice. The BABL/c mice were randomly divided into two groups: The Eps8 group and the PBS group, with 8 healthy mice included in each experimental group. The animals were immunized subcutaneously once a week for three weeks; the mice received $100 \mu \mathrm{g}$ of either Eps8 protein or PBS mixed with complete
Freund's adjuvant for the first immunization, and for the second and third immunizations, the same quantity of either Eps8 protein or PBS was mixed with incomplete Freund's adjuvant. Seven days subsequent to the third immunization, the mice were inoculated subcutaneously with $7 \times 10^{6} 4 \mathrm{~T} 1$ tumor cells in the right armpit. The mice were monitored each day and the day of the tumor cell inoculation was recorded as day 0 .

In vivo antitumor experiments. The animals were vaccinated and then challenged with tumor cells. The antitumor effects were evaluated by measuring the tumor volume, tumor growth inhibition rate and overall survival time. In the tumor protection experiments, the tumor size was determined by the measurement of the shortest (a) and longest (b) diameter using vernier calipers, every 3 days. The volume $(\mathrm{V})$ of the tumors was calculated using the formula: $V=\left(a^{2} b / 2\right)(26)$. For the comparison of tumor growth inhibition rates, mice were sacrificed by neck dislocation 28 days following inoculation. The tumors were surgically removed and weighed. The inhibition rate was calculated as follows: Inhibition rate $(\%)=(1-$ tumor weight of Eps8 group/tumor weight of PBS group) x 100 . For survival analysis, an observation period of 60 days was adopted following the inoculation of the mice with 4T1 tumor cells.

Flow cytometric analysis. BALB/c mice were grouped and treated as described previously, and sacrificed 28 days following inoculation with tumor cells. The splenocytes were collected as described previously, and incubated with the corresponding antibodies: Fluorescein isothiocyanate (FITC) anti-mouse CD4 $\mathrm{mAb}$ and PerCP anti-mouse CD8 $\mathrm{mAb}$ (BioLegend). The negative control consisted of splenocytes labeled with the corresponding isotype control antibodies (27). The Mouse Regulatory T cell Staining kit (eBiosciences, Inc., San Diego, CA, USA) was used for detecting the $\mathrm{CD} 4^{+} \mathrm{CD} 25^{+}$ FoxP $3^{+}$Treg cells among the splenocytes. Briefly, the splenocytes of the BALB/c mice were surface-stained with FITC anti-mouse CD4 mAb and APC anti-mouse CD25 mAb. Subsequently, the cells were permeabilized using the FoxP3 Staining Buffer set, and stained with rat IgG2a kappa isotype control PE or anti-mouse/rat Foxp3 PE. All samples were run on a FACSCalibur flow cytometer (Becton-Dickinson) and data were analyzed using WinMDI 2.9 software (Joseph Trotter, The Scripps Institute, La Jolla, CA, USA).

Cytotoxic T lymphocyte (CTL) assay. The splenocytes were harvested from the tumor-bearing mice that had been immunized with vaccines and cultured as described previously. The viable splenocytes were regarded as the effector cells and the 4T1 cells were used as the target cells. The cytotoxic effect of the splenocytes against the 4T1 cells was measured by the lactate dehydrogenase (LDH) assay and calculated as described previously.

Statistical analysis. Data are presented as the mean \pm standard deviation. The significance of the statistical comparisons was calculated by an analysis of variance (ANOVA) or the Student's t-test using SPSS software, version 13.0 (SPSS, Inc., Chicago, IL, USA). The comparison of the survival data of the two groups was evaluated with a log-rank test of the Kaplan-Meier 
survival curves. The tumor volumes were analyzed using a repeated measures ANOVA. $\mathrm{P}<0.05$ was considered to indicate a statistically significant difference.

\section{Results}

Preparation and identification of Eps8. The PCR products were electrophoresed on $1 \%$ agarose gels and visualized by ethidium bromide staining under UV light. The results revealed that the fragment was $\sim 801 \mathrm{bp}$, as expected (Fig. 1A, lanes 1 and 2). Following purification, the Eps8 protein was expressed on the gel with a purity of $90 \%$ in the total proteins (Fig. 1B, lanes 4 and 5). In addition, a western blot assay confirmed that the expression product had the antigenicity of Eps8 (Fig. 1C, lanes 1-3).

Changes in the phenotype of DCs following pulsation with Eps8. The DCs were generated from mouse bone marrow cells. On day 7 , either TNF- $\alpha$, Eps 8 or OVA were added to the DCs, to induce maturation. Following 8 days of cultivation, unpulsed DCs (the DC group), OVA-pulsed DCs (the DC + OVA group) and Eps8-pulsed DCs (the DC + Eps8 group) were collected and analyzed by flow cytometry (Fig. 2). Compared with the DC and the DC + OVA groups, significant increases in the expression of MHC-II, CD80 and CD86 were observed in the $\mathrm{DC}+$ Eps8 group $(\mathrm{P}<0.05)$; however, there was no significant difference in CD11c expression among these three groups $(\mathrm{P}>0.05)$.

Eps8 promotes DC maturation, T-cell proliferation and cytotoxicity in vitro. The results demonstrated that Eps8 stimulated the maturation of DCs, and their secretion of IL-12 p70. The IL-12 p70 level peaked at day 3 in all groups of DCs; the highest level was achieved by the DC + Eps8 group, and was significantly higher than the levels observed in the remaining two (control) groups (Fig. 3C). IFN- $\gamma$ is a significant effector cytokine released by $\mathrm{T}$ cells. The ELISA results revealed that Eps8-pulsed DCs enhanced the IFN- $\gamma$ secretion by the splenocytes; the concentration of IFN- $\gamma$ was significantly greater in this group, compared with that of the two control groups (Fig. 3D).

The proliferative ability and the cytotoxicity of the splenocytes were also increased by Eps8. The data demonstrated an enhanced proliferation rate (by 3.28 \pm 0.34 -fold) of splenocytes co-cultured with Eps8-pulsed DCs (Fig. 3A). As demonstrated in Fig. 3B, at an E/T cell ratio of 5:1, the splenocytes that were co-cultured with Eps8-pulsed DCs were able to lyse $54.89 \pm 4.99 \%$ of the target cells, while the other two (control) groups of splenocytes only exhibited a background lysis of 4T1 cells. In summary, Eps8 possessed the ability to stimulate DC and T-cell activities in vitro.

Prophylactic antitumor effect of the Eps8 vaccine in the murine $4 T 1$ breast cancer model. The 4T1 breast cancer model was used to investigate the effect of the Eps8 vaccine on tumor growth in vivo. The results demonstrated that none $(0 \%)$ of the mice remained tumor free; however, the tumor growth in the Eps8-immunized mice was inhibited significantly compared with that of the PBS control mice (Fig. 4A). The tumor weights were measured when the mice were sacrificed and the mean

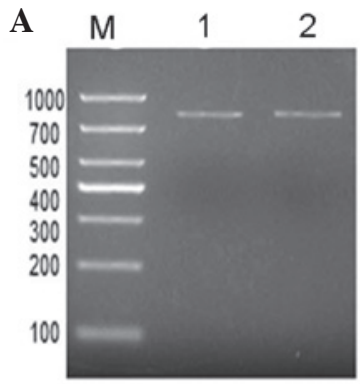

B $\quad M \quad M \quad 1 \quad 2 \quad 3 \quad 4 \quad 5$

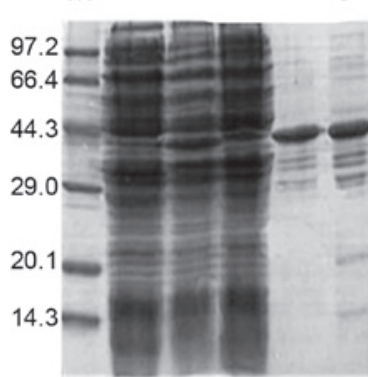

C

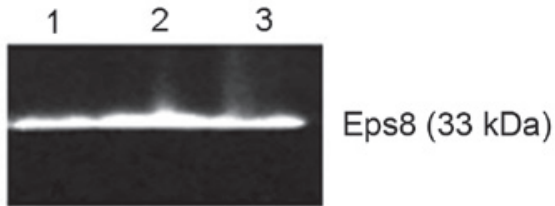

Figure 1. Agarose electrophoresis, SDS-PAGE and western blot analysis of the expression product of the recombinant plasmid pET28a-Eps8 (A) Agarose electrophoresis of the RT-PCR products of mouse Eps8 cDNA. Lanes 1 and 2, RT-PCR product of Eps8; lane M, DNA ladder marker. (B) SDS-PAGE of the different samples followed by Coomassie blue staining of the gel. Lane 1, eluent of the BL21 strain carrying pET28a-Eps8; lane 2, supernatants of the BL21 strain carrying Eps8 following induction by IPTG; lane 3, supernatants of the BL21 strain carrying Eps8 prior to induction by IPTG; lanes 4 and 5, proteins purified from the BL21 strain carrying pET28aEps8; lane M, molecular weight protein markers. (C) Western blot analysis with an anti-Eps8 monoclonal antibody. Lanes 1-3, expression product of the recombinant plasmid pET28a-Eps8 in BL21 E.coli cells. Eps8, epidermal growth factor receptor pathway substrate 8 .

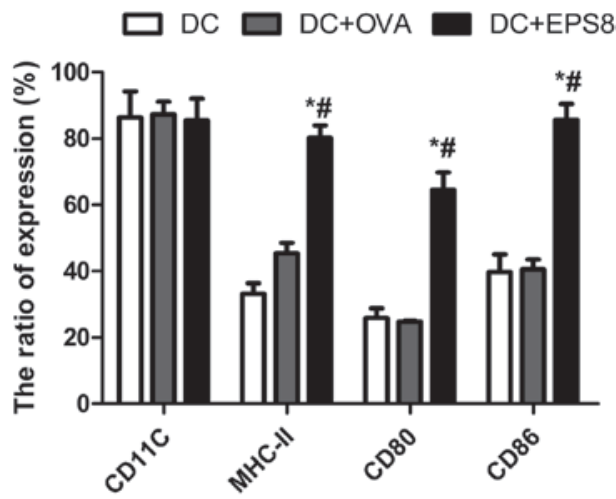

Figure 2. Flow cytometric analysis of DCs. In the DC group, the expression rates of the antigen-presenting molecule, MHC II, and the co-stimulatory molecules, CD80 and CD86, were 33.22 $\pm 3.18,25.88 \pm 2.94$ and 39.69 $\pm 5.35 \%$, respectively. In the DC + OVA group, the expression rates were $45.38 \pm 3.17$, $24.82 \pm 0.26$ and $40.53 \pm 2.98 \%$, respectively; in the DC + Eps8 group, the expression rates were $80.19 \pm 3.78,64.63 \pm 4.83$ and $85.63 \pm 4.83 \%$, respectively. ${ }^{*} \mathrm{P}<0.05$ vs. the $\mathrm{DC}$ group and ${ }^{\#} \mathrm{P}<0.05$ vs. $\mathrm{DC}+\mathrm{OVA}$ group. $\mathrm{DC}$, dendritic cell; OVA, ovalbumin; DC, unpulsed DCs; DC + OVA, DCs pulsed with OVA protein; DC + Eps8, DCs pulsed with Eps8 protein; MHC II, major histocompatibility complex class II.

tumor weight of the Eps8 group was significantly lower than that of the PBS group (Fig. 4B). In addition, the inhibition rate of tumor growth was calculated to be $33.23 \%$. To determine the immune protection effect of the Eps8 vaccine, mice were inoculated subcutaneously with 4T1 cells following immunization, and the survival time was measured. The results revealed that all mice in the two groups succumbed within 55 days of 

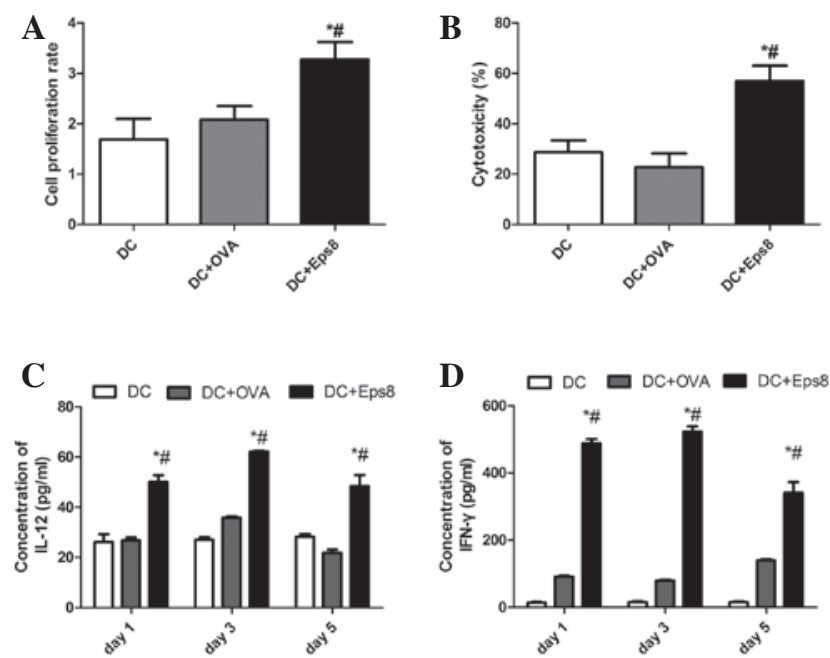

Figure 3. Eps8 protein stimulates DC and T-cell activities in vitro. (A) The proliferation rate of splenocytes co-cultured with DCs. The splenocytes were co-cultured with different DCs for 3 days at a stimulator to responder ratio of 1:5. The proliferation rate was significantly higher in the DC + Eps8 group compared with all other groups $(\mathrm{P}<0.01)$, while no significant differences were observed between the DC + OVA group and the DC group. The results are representative of three independent experiments. (B) Cytotoxic activity of splenocytes against 4T1 breast tumor cells. The splenocytes were co-cultured with different DCs, and incubated with 4T1 cells for $24 \mathrm{~h}$ at an effector to target ratio (E:T) of 20:1. The CTL activity of the splenocytes was significantly increased in the DC + Eps8 group compared with that of the OVA + DC and DC groups $(\mathrm{P}<0.01)$. Results are representative of three independent experiments. (C) Analysis of the level of IL-12 secreted by the DCs. The concentration of IL-12 in the supernatant of cultured DCs was analyzed by ELISA assay on days 1,3 and 5 . Eps 8 protein $(50 \mathrm{ng} / \mathrm{ml})$ stimulated DCs secreted the highest level of IL-12 on day 3, and the concentration of IL-12 in the DC + Eps8 group was significantly higher than that of the DC + OVA and DC groups at all time points. (D) Regulation of T-cell function. The splenocytes were co-cultured with different DCs for 5 days. The supernatants were collected at different time points and cytokine production of IFN- $\gamma$ was measured by ELISA assay. The levels of IFN- $\gamma$ secreted by the DC + Eps8 group were significantly higher than the other two groups on days 1,3 and 5 $(\mathrm{P}<0.01)$. Results are representative of three independent experiments. ${ }^{*} \mathrm{P}<0.05$ vs. the DC group and ${ }^{~} \mathrm{P}<0.05$ vs. the DC + OVA group. Eps8, epidermal growth factor receptor pathway substrate 8; OVA, ovalbumin; DC, dendritic cell; DC, unpulsed DCs; DC + OVA, DCs pulsed with OVA protein; DC + Eps8, DCs plused with Eps8 protein; CTL, cytotoxic T lymphocyte; IL, interleukin; ELISA, enzyme-linked immunosorbent assay.

the inoculation, but the survival time of the tumor-bearing mice that had been immunized with Eps8 was significantly longer than that of the PBS group (Fig. 4C and D). These results suggested that treatment with Eps8 prior to tumor cell inoculation resulted in a significant prophylactic antitumor effect in the 4T1 breast cancer model.

Eps8 vaccination increases the cytotoxicity of splenocytes in tumor-bearing mice. CTL activity was induced by immunization with Eps8. The LDH assay was performed at E/T cell ratios of 10:1, 20:1 and 40:1. T cells isolated from the spleens of the tumor-bearing mice pre-treated with the Eps8 vaccine exhibited greater cytotoxicity against 4T1 cells than those of the PBS group, when the E/T cell ratios were 20:1 and 40:1 (Fig. 5). The results indicated that the killing activity observed may have resulted from Eps8-specific cellular immune responses, which contributed to the observed in vivo antitumor immunity.
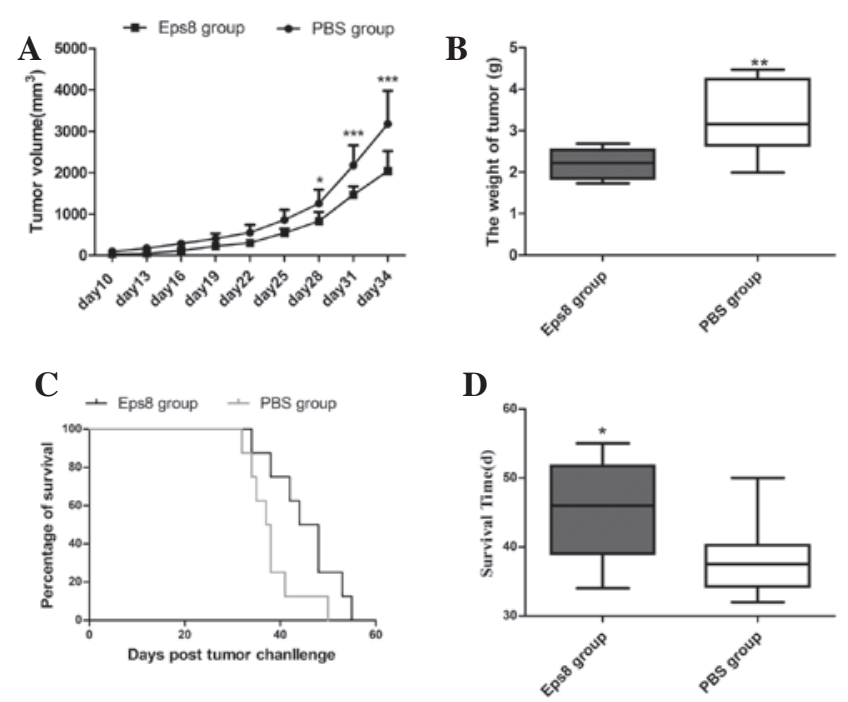

Figure 4. Eps8 protein vaccine inhibited tumor growth in vivo. Mice were vaccinated three times with the Eps8 protein or PBS at weekly intervals prior to tumor implantation. (A) Growth of 4T1 tumors in mice treated with vaccines. The tumor volume was monitored every 3 days following the inoculation with 4T1 tumor cells. The volume of the tumors in the Eps8 group was significantly smaller compared with that of the PBS group on days 28, 31 and 34, following the tumor challenge. (B) The mean tumor weight of the Eps8 group was significantly lower compared with that of the PBS group on day $28(\mathrm{P}<0.01)$. (C) All mice died within 55 days of inoculation with 4T1 tumor cells. The Kaplan-Meier survival curves demonstrate superior protection from tumor outgrowth by the Eps8 vaccine. (D) The survival time of mice in the Eps8 group was prolonged, compared with that of the PBS group. $(\mathrm{P}<0.05) .\left({ }^{*} \mathrm{P}<0.05,{ }^{* *} \mathrm{P}<0.01\right.$ and $\left.{ }^{* * *} \mathrm{P}<0.001\right)$. Eps8, epidermal growth factor receptor pathway substrate 8 ; PBS, phosphate-buffered saline.

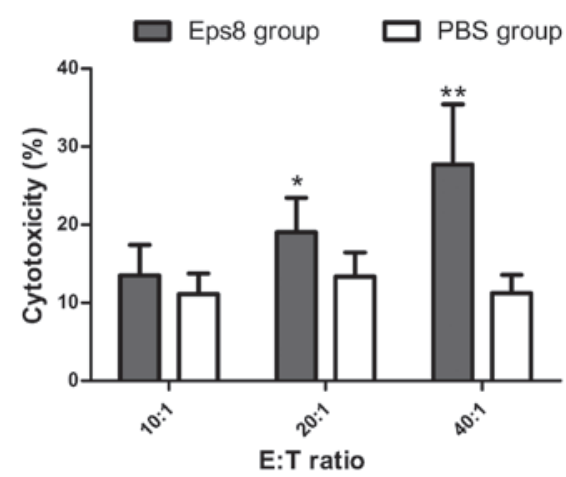

Figure 5. Eps8 protein vaccine promoted CTL cytotoxicity of the splenocytes of the tumor-bearing mice. Mice were vaccinated three times with the Eps8 protein or PBS at one-week intervals, prior to inoculation with 4T1 tumor cells. The Eps8 group demonstrated higher CTL cytotoxicity than the PBS group at E:T ratios of 20:1 and 40:1. Results are representative of three independent experiments. ${ }^{*} \mathrm{P}<0.05$ and ${ }^{* *} \mathrm{P}<0.01$. Eps8, epidermal growth factor receptor pathway substrate 8 ; CTL, cytotoxic T lymphocyte; PBS, phosphatebuffered saline; E:T, effector to target.

Changes in the immune cell population with Eps8 vaccination. The proportions of splenocyte subsets were detected by flow cytometry. Eps 8 immunization increased the $\mathrm{CD}^{+} / \mathrm{CD}^{+} \mathrm{T}$ cell ratio compared with that of the PBS control mice (Fig. 6A). The proportion of $\mathrm{CD} 4^{+} \mathrm{CD} 25^{+}$FoxP3 ${ }^{+}$Treg cells in the tumor-bearing mice immunized with the Eps8 vaccine or injected with PBS was also measured by FACS. Cells in the lymphocyte gate were used for analysis and the 
A

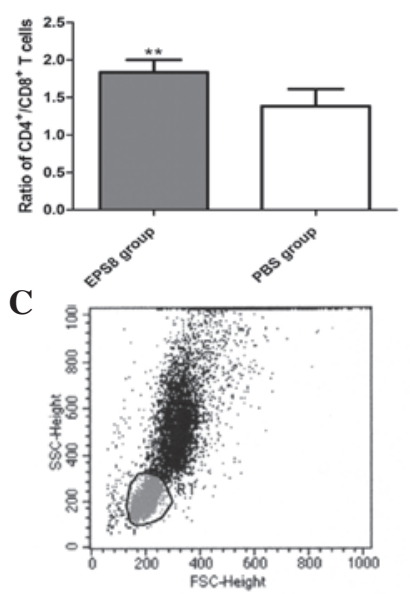

D

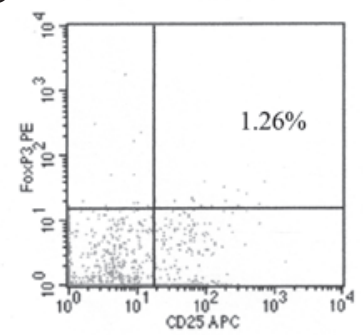

$\mathbf{E}$

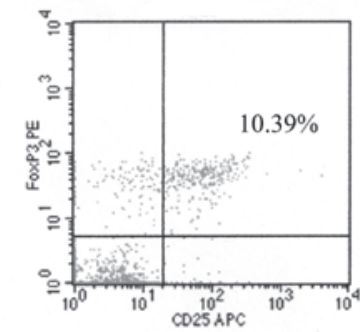

B
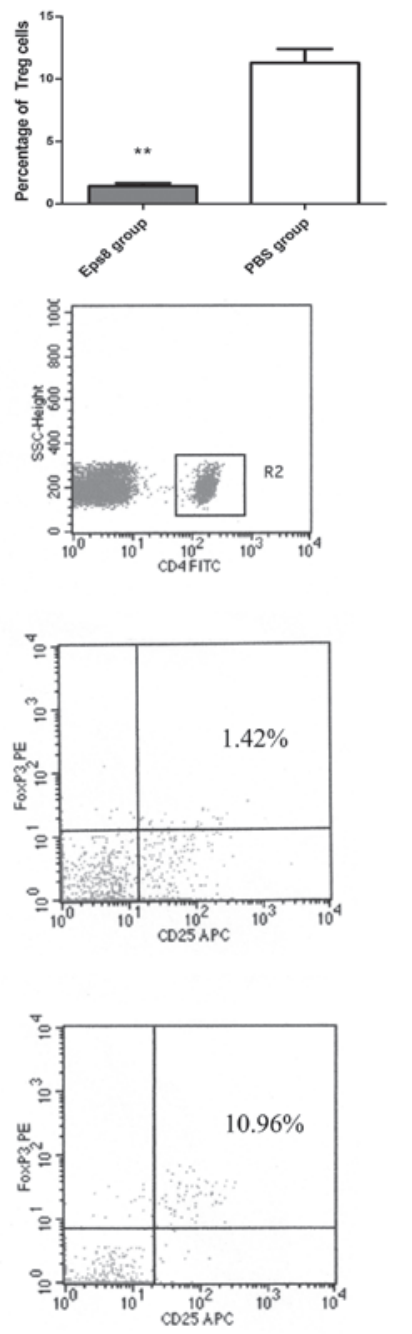

Figure 6. Effect of the Eps8 protein vaccination on immune cell populations. The mice were vaccinated three times with either the Eps8 protein or PBS prior to being inoculated with $4 \mathrm{~T} 1$ tumor cells. The splenocytes from tumor-bearing mice were analyzed for the $\mathrm{CD} 4^{+} / \mathrm{CD}^{+} \mathrm{T}$ cell ratio and the percentage of Treg cells, by flow cytometry. Results are representative of three independent experiments. (A) Splenocytes were collected as described in Materials and methods, and the proportions of the subsets of splenocytes were analyzed. The $\mathrm{CD} 4^{+} / \mathrm{CD}^{+} \mathrm{T}$ cell ratio was increased in the Eps 8 group $(\mathrm{P}<0.01)$. (B) The percentage of $\mathrm{CD}^{+} \mathrm{CD} 25^{+} \mathrm{FoxP} 3^{+}$Treg cells was identified to be significantly lower in the Eps8 group compared with that of the PBS group $(\mathrm{P}<0.01)$. (C) Cells in the lymphocyte gate were used for analysis, and a CD4 gating strategy was used as a secondary gating method. (D) Representative results of $\mathrm{CD} 4^{+} \mathrm{CD} 25^{+} \mathrm{FoxP} 3^{+}$Treg cells of mice in (D) the Eps8 group and (E) the PBS group. ${ }^{*} \mathrm{P}<0.05$ and ${ }^{* *} \mathrm{P}<0.01$. Eps8, epidermal growth factor receptor pathway substrate 8; PBS, phosphate-buffered saline; Treg, regulatory $\mathrm{T}$ cell.

CD4 gating strategy was used as a secondary gating method (Fig. 6C). As demonstrated in Fig. 6B, the percentage of $\mathrm{CD} 4{ }^{+} \mathrm{CD} 25^{+} \mathrm{FoxP}^{+}$Treg cells significantly decreased in the splenocytes of the Eps8-vaccinated mice compared with that of the PBS control mice. Representative results are shown in Fig. 6D and E.

\section{Discussion}

Eps8 is a significant tumor-associated antigen with restricted expression and is the intracellular receptor of EGFR (28).

Certain studies have suggested that the intracellular domain (ICD) of proteins may be more immunogenic than the extracellular domain (ECD), as the ICD is not usually exposed to the immune system. Antibodies binding to the ICD presented in a tumor bed or shed by dying cells may contribute to increases in the cellular immune response, by inducing antibody-dependent cell-mediated cytotoxicity $(29,30)$. In the present study, Eps8 protein exerted a significant antibody response and the antibody titer was increased with each immunization (data not shown). Such unique characteristics make Eps8 a candidate target antigen for antitumor vaccines.

In this study, the Eps8 protein vaccine induced significant T-cell proliferation along with cytotoxic activity towards the Eps8-expressing 4T1 tumor cells (Fig. 3). Treatment of the immature DCs with Eps8 induced an increased expression of co-stimulatory molecules, including CD80, CD86 and MHC-II (Fig. 2). DCs are able to process antigenic material and present it on their surface to other cells of the immune system; therefore, they play a significant role in the antitumor effect by processing or carrying tumor-associated antigens (31-33). A study by Banchereau et al demonstrated that immature DCs are better equipped for capturing antigen and migrating to the lymph nodes than mature DCs $(19,31)$. However, mature DCs are superior in representing antigens and stimulating T cells compared with immature DCs (19). A suggested mechanism for the antitumor effect of the Eps8 protein may include increased maturation of the DCs and increased cytokine secretion, which further activates the $\mathrm{T}$ cells.

Due to the results in vitro, we investigated the potential use of Eps8 as a breast cancer vaccine in vivo. The Eps8 protein vaccine promoted cytotoxic T-cell activity towards Eps8-expressing targets. Immune responses induced by the Eps8 protein inhibited tumor growth in the murine 4T1 breast cancer model. In order to investigate the mechanism of action of the prophylactic Eps8 vaccination, we measured the proportion of $\mathrm{CD} 4^{+} \mathrm{CD} 25^{+}$Treg cells in $\mathrm{CD} 4^{+} \mathrm{T}$ cells, in Eps8 vaccinated mice. $\mathrm{CD} 4{ }^{+} \mathrm{CD} 25^{+}$Treg populations, originally demonstrated to suppress autoimmune responses, are also crucial in controlling antitumor immune responses (34). Naturally occurring regulatory $\mathrm{T}$ cells $\left(\mathrm{CD} 4{ }^{+} \mathrm{CD} 25^{+} \mathrm{T}\right.$ cells), are important in tumor invasion and the downregulation of immune responses against established tumors (35). Although tumors themselves are potent inducers of Treg activity, the currently used cancer vaccination schemes may also lead to enhanced frequencies of Treg cells, characterized by a surface $\mathrm{CD} 4{ }^{+} \mathrm{CD} 25^{\text {high }}$ phenotype and intracellular expression of FOXP3 $(36,37)$. Failure of host antitumor immunity may be caused by exaggerated suppression of tumor-associated antigen-reactive CTLs by Treg cells, which contributes to the growth of human tumors in vivo. Treg cells are also associated with a high risk of mortality and a reduced survival rate. Thus, blocking Treg cells may be a therapeutic target in numerous types of cancer (36). Our results revealed that mice immunized with Eps8 exhibited decreased ratios of $\mathrm{CD} 4^{+} \mathrm{CD} 25^{+}$Treg cells to $\mathrm{CD} 4^{+} \mathrm{T}$ cells, which may in turn assist in the prevention of tumor outgrowth and prolong the survival of the challenged mice.

To the best of our knowledge, our study is the first to demonstrate that an Eps8 vaccination is able to provide 
prophylaxis against breast cancer, in a 4T1 model system. Therefore, our results may serve as a rationale for developing a novel and effective vaccine against human breast cancer in the future.

\section{Acknowledgements}

The authors would like to thank Dr Yanqing Ding for providing the MA782 mouse breast cancer cell line, and Ms. Lin Lou and Ms. Miaoxia Li for their expert administrative assistance. This study was supported by the Key Program of Natural Science Foundation of Guangdong Province, China (grant no. 9251051501000007$)$ and the Program for New Century Excellent Talents in University, Ministry of Education of China (grant no. NCET-09-0087).

\section{References}

1. Parkin DM, Bray F, Ferlay J and Pisani P: Estimating the world cancer burden: Globocan 2000. Int J Cancer 94: 153-156, 2001.

2. Lanari C, Wargon V, Rojas P and Molinolo AA: Antiprogestins in breast cancer treatment: are we ready? Endocr Relat Cancer 19 R35-50, 2012.

3. Fletcher SW: Breast cancer screening: a 35-year perspective. Epidemiol Rev 33: 165-175, 2011.

4. Esteva FJ: Monoclonal antibodies, small molecules, and vaccines in the treatment of breast cancer. Oncologist 9 (Suppl 3): S4-S9, 2004

5. Fioretti D, Iurescia S, Fazio VM and Rinaldi M: DNA vaccines: developing new strategies against cancer. J Biomed Biotechnol 2010: 174378, 2010.

6. Peoples GE, Gurney JM, Hueman MT, et al: Clinical trial results of a HER2/neu (E75) vaccine to prevent recurrence in high-risk breast cancer patients. J Clin Oncol 23: 7536-7545, 2005.

7. Mohebtash M, Tsang KY, Madan RA, et al: A pilot study of MUC-1/CEA/TRICOM poxviral-based vaccine in patients with metastatic breast and ovarian cancer. Clin Cancer Res 17: 7164-7173, 2011

8. Emens LA, Reilly RT and Jaffee EM: Breast cancer vaccines: maximizing cancer treatment by tapping into host immunity. Endocr Relat Cancer 12: 1-17, 2005.

9. Curigliano G, Spitaleri G, Pietri E, et al: Breast cancer vaccines: a clinical reality or fairy tale? Ann Oncol 17: 750-762, 2006.

10. Offenhäeuser N, Borgonovo A, Disanza A, Romano P, Ponzanelli I and Iannolo G: The eps8 family of proteins links growth factor stimulation to actin reorganization generating functional redundancy in the Ras/Rac pathway. Mol. Biol Cell 15: 91-98, 2004.

11. Fazioli F, Minichiello L, Matoska V, Castagnino P, Miki T, Wong WT and Di Fiore PP: Eps8, a substrate for the epidermal growth factor receptor kinase, enhances EGF-dependent mitogenic signals. EMBO J 12: 3799-3808, 1993.

12. Yap LF, Jenei V, Robinson CM, et al: Upregulation of Eps8 in oral squamous cell carcinoma promotes cell migration and invasion through integrin-dependent Rac1 activation. Oncogene 28: 25242534, 2009.

13. Chen YJ, Shen MR, Chen YJ, Maa MC and Leu TH: Eps8 decreases chemosensitivity and affects survival of cervical cancer patients. Mol Cancer Ther 7: 1376-1385, 2008.

14. Maa MC, Lee JC, Chen YJ, et al: Eps8 facilitates cellular growth and motility of colon cancer cells by increasing the expression and activity of focal adhesion kinase. J Biol Chem 282: 19399-19409, 2007.

15. Welsch T, Endlich K, Giese T, Büchler MW and Schmidt J: Eps8 is increased in pancreatic cancer and required for dynamic actin-based cell protrusions and intercellular cytoskeletal organization. Cancer Lett 255: 205-218, 2007.

16. Wang H, Patel V, Miyazaki H, Gutkind JS and Yeudall WA: Role for EPS8 in squamous carcinogenesis. Carcinogenesis 30 : $165-174,2009$
17. Xu M, Shorts-Cary L, Knox AJ, Kleinsmidt-DeMasters B, Lillehei K and Wierman ME: Epidermal growth factor receptor pathway substrate 8 is overexpressed in human pituitary tumors: role in proliferation and survival. Endocrinology 150: 2064-2071, 2009.

18. Bashir M, Kirmani D, Bhat HF, et al: P66shc and its downstream Eps8 and Rac1 proteins are upregulated in esophageal cancers. Cell Commun Signal 8: 13-19, 2010.

19. Matoskova B, Wong WT, Salcini AE, Pelicci PG and Di Fiore PP: Constitutive phosphorylation of eps8 in tumor cell lines: relevance to malignant transformation. Mol Cell Biol 15: 3805-3812, 1995.

20. Funato Y, Terabayashi T, Suenaga N, Seiki M, Takenawa T and Miki H: IRSp53/Eps8 complex is important for positive regulation of Rac and cancer cell motility/invasiveness. Cancer Res 64: 5237-5244, 2004.

21. Wang H, Teh MT, Ji Y, et al: EPS8 upregulates FOXM1 expression, enhancing cell growth and motility. Carcinogenesis 31: 1132$1141,2010$.

22. Terme M, Tomasello E, Maruyama K, et al: IL-4 confers NK stimulatory capacity to murine dendritic cells: a signaling pathway involving KARAP/DAP12-triggering receptor expressed on myeloid cell 2 molecules. J Immunol 172: 5957-5966, 2004.

23. Matheu MP, Sen D, Cahalan MD and Parker I: Generation of bone marrow derived murine dendritic cells for use in 2-photon imaging. J Vis Exp 17: 773-776, 2008.

24. Liu J, Schmidt CS, Zhao F, et al: LIGHT-deficiency impairs $\mathrm{CD} 8+\mathrm{T}$ cell expansion, but not effector function. Int Immunol 5: 861-870, 2003

25. Mosmann T: Rapid colorimetric assay for cellular growth and survival: application to proliferation and cytotoxicity assays. J Immunol Methods 65: 55-63, 1983.

26. Park HJ, Ahn KJ, Ahn SD, et al: Susceptibility of cancer cells to beta-lapachone is enhanced by ionizing radiation. Int $\mathrm{J}$ Radiat Oncol Biol Phys 61: 212-219, 2005.

27. Märten A, Renoth S, von Lilienfeld-Toal M, et al: Enhanced lytic activity of cytokine-induced killer cells against multiple myeloma cells after co-culture with idiotype-pulsed dendritic cells. Haematologica 86: 1029-1037, 2001.

28. Burke P, Schooler K and Wiley HS: Regulation of epidermal growth factor receptor signaling by endocytosis and intracellular trafficking. Mol Biol Cell 12: 1897-1910, 2001.

29. Disis ML, Schiffman K, Guthrie K, et al: Effect of dose on immune response in patients vaccinated with an her-2/neu intracellular domain protein-based vaccine. J Clin Oncol 22: 1916-1925, 2004.

30. Foy TM, Bannink J, Sutherland RA, et al: Vaccination with Her-2/neu DNA or protein subunits protects against growth of a Her-2/neu expressing murine tumor. Vaccine 19: 2598-2606, 2001.

31. Banchereau J, and Steinman RM: Dendritic cells and the control of immunity. Nature 392: 245-252, 1998.

32. Santegoets SJ, van den Eertwegh AJ, van de Loosdrecht AA, Scheper RJ and de Gruijl TD: Human dendritic cell line models for DC differentiation and clinical DC vaccination studies. J Leukoc Biol 84: 1364-1373, 2008.

33. Morse MA, Chui S, Hobeika A, Lyerly HK and Clay T: Recent developments in therapeutic cancer vaccines. Nat Clin Pract Oncol 2: 108-113, 2005.

34. Nishikawa H, Kato T, Hirayama M, et al: Regulatory $\mathrm{T}$ Cell-resistant CD8+ T cells induced by glucocorticoidinduced tumor necrosis factor receptor signaling. Cancer Res 68 : 5948-5954, 2008.

35. Akbari A and Rezaei A: In vitro selective depletion of CD4(+) CD25(+) regulatory T-cells from PBMC using anti-tac-SAP. J Immunotoxicol 9: 368-373, 2012.

36. Curiel TJ, Coukos G, Zou L, et al: Specific recruitment of regulatory $\mathrm{T}$ cells in ovarian carcinoma fosters immune privilege and predicts reduced survival. Nat Med 10: 942-949, 2004.

37. Banerjee DK, Dhodapkar MV, Matayeva E, Steinman RM and Dhodapkar KM: Expansion of FOXP3high regulatory T cells by human dendritic cells (DCs) in vitro and after injection of cytokine-matured DCs in myeloma patients. Blood 108: $2655-2661,2006$ 